\title{
Colecistopatia aguda e crônica: análise comparativa das taxas e causas de conversão para laparotomia
}

\section{Acute and chronic cholecystopathies: analysis comparative of the rates and causes of conversion to laparotomy}

luiz Carlos Von Bahten, TCBC-PR'; Ana Cristina Isa, AsCBC-PR²; Pâmela Concelção Figueiredo, AsCBC-PR²; Hugo Campezato³; Rosana Hapsi Isa ${ }^{3}$; Márcia Olandoski ${ }^{4}$

\author{
RE S U M O
}

\begin{abstract}
Objetivo: Analisar a taxa e as principais causas de conversão de colecistectomia videolaparoscópica para cirurgia aberta entre colecistites agudas e crônicas. Métodos: Estudo retrospectivo, analisando 1359 prontuários de pacientes submetidos à colecistectomia no Serviço de Cirurgia Geral do Hospital Universitário Cajuru no período de janeiro de 2000 à outubro de 2006. Resultados: Realizaram-se 1066 colecistectomias videolaparoscópicas, sendo, 701(65,75\%) por colecistopatia crônica calculosa, 356 (33,39\%) por colecistopatia aguda calculosa, sete $(0,65 \%)$ por pólipos de vesícula, duas $(0,21 \%)$ por vesícula hidrópica. A taxa de conversão na colecistopatia aguda foi de 7,86\%, e na colecistite crônica 2,85\% ( $p=0,0003)$. A média de idade das taxas de conversão foi de 50,96 $\pm 17,49$ anos para colecistopatia aguda e de $56,45 \pm 12,28$ anos para crônica $(p=0,234)$. O tempo cirúrgico mediano foi de 152,5 (30 - 36) minutos para aguda e 157,5 (90 - 240) para crônica ( $p=0,959)$. As principais causas de conversão nas colecistopatias crônicas foram: anatomia obscura (16 pacientes) e aderências (14 pacientes), sendo que em 10 casos as duas causas estavam associadas. Já na forma aguda, as principais causas foram aderência (13 pacientes) e dificuldades técnicas (8 pacientes); com diferença significativa $(p=0,008)$. Conclusão: A taxa de conversão de cirurgia videolaparoscópica para cirurgia aberta é maior nos casos de colecistopatia calculosa aguda do que na crônica. Nesta, o fator que mais dificultou a realização do procedimento videolaparoscópico em nosso serviço foi a alteração anatômica; já naquela, foi a presença de aderências.
\end{abstract}

Descritores: Colecistectomia. Colecistite aguda. Doença crônica.

\section{INTRODUÇÃO}

A colecistectomia videolaparoscópica é o tratamento de escolha para a colecistopatia calculosa. No entanto, há uma elevada proporção de casos em que não é possível sua realização. Além disso, em alguns pacientes, quando se tem dificuldades técnicas, torna-se necessário a conversão de cirurgia videolaparoscópica para cirurgia aberta.

Estudos mostraram que esta conversão é feita em 1,5 a 19\% dos pacientes devido, principalmente, à dificuldade anatômica e complicações inerentes à dissecação laparoscópica ${ }^{1-5}$.

Um fato comum observado em estudos que analisaram a taxa de conversão cirúrgica em colecistopatias é o predomínio desta taxa em colecistite aguda. Além disso, a principal causa que leva à essa conversão é a dificuldade de identificação das estruturas anatômicas e dissecção do triângulo de Calot, ocasionada por inflamação intensa e aderências ${ }^{6-13}$.

O objetivo deste trabalho é a compreensão das complicações da cirurgia laparoscópica bem como as causas de conversão para o método convencional e o conhecimento das particularidades entre a colecistopatia aguda e crônica, de suma importância para que se possa atuar preventivamente, reduzindo a morbidade decorrente desse procedimento cirúrgico.

\section{MÉTODOS}

Analisar 1359 prontuários de pacientes submetidos à colecistectomia no serviço de Cirurgia Geral do Hospital Universitário Cajuru no período de 2000 a 2006, utilizando um protocolo elaborado pelo próprio grupo de pesquisa.

Destes prontuários observaram-se dados epidemiológicos referentes ao sexo, idade, presença de comorbidades, dados pré-operatórios concernentes à presença de cirurgia abdominal prévia, causas da colecistectomia e tempo de evolução dos sintomas. Em relação ao período operatório, estudou-se a causa da conversão, tempo de operação e achados da colangiografia per - operatória.

Os resultados obtidos no estudo foram expressos por frequências e percentuais ou por médias, desvios padrões, medianas, valores mínimos e máximos. Para a comparação dos grupos de pacientes com colecistopatia aguda 
e crônica em relação a variáveis categóricas foi usado o teste de Qui-quadrado ou o teste exato de Fisher. Essas comparações em relação a variáveis quantitativas foram feitas usando-se o teste t de Student para amostras independentes ou o teste não-paramétrico de Mann-Whitney, quando apropriado. Valores de $p<0,05$ indicaram significância estatística.

\section{RESULTADOS}

Foram analisados 1359 prontuários de pacientes submetidos à colecistectomia, 1066 por via videolaparoscópica. Destes, 701(65,75\%) foram por colecistopatia crônica calculosa, 356 (33,39\%) colecistopatia aguda calculosa, sete $(0,65 \%)$ por pólipos de vesícula e dois $(0,21 \%)$ casos por vesícula hidrópica. A conversão para cirurgia aberta foi verificada em 48 (4,5\%) pacientes. Destes casos, as indicações cirúrgicas foram de colecistite aguda em 28 pacientes, correspondendo a uma taxa de conversão de $7,86 \%$ entre as causas agudas de cirurgia videolaparoscópica; e de colecistite crônica em 20 pacientes, correspondendo a uma taxa de conversão de 2,85\% entre as causas crônicas de cirurgia videolaparoscópica, havendo diferença significativa $(p=0,0003)$.

Dos pacientes com colecistite crônica, 14 (70\%) foram do sexo feminino e sete do masculino. Já na aguda, $18(64,28 \%)$ pacientes foram do sexo feminino e nove do masculino, sem diferença significativa $(p=0,762)$. A média de idade das taxas de conversão foi de 50,96 $\pm 17,49$ anos para aguda e de 56,45 $\pm 12,28$ anos para a colecistite crônica, sem diferença significativa $(p=0,234)$.

Em pacientes submetidos à colecistectomia videolaparoscópica verificaram-se cirurgia abdominal prévia em 11 (55\%) casos nas causas crônicas (Limite de 95\% de confiança de 40,93\% a 69,07\%) e em 12 (42,85\%) casos na forma aguda (Limite de $95 \%$ de confiança para esta proporção é de $28,86 \%$ a 56,86\%). Já em relação aos dias de internamento, a mediana foi de 6,5 (2 - 16) para colecistopatia aguda e 8,5 (3 - 68) dias para a crônica, sem diferença significativa $(p=0,159)$. O tempo cirúrgico mediano foi de 152,5 (30 - 36) minutos para colecistopatia aguda e 157,5 (90 - 240) para colecistopatia crônica, sem diferença significativa ( $p=0,959)$.

Em relação às taxas de conversão cirúrgica em colecistopatia aguda, as principais causas foram aderência (13 pacientes), seguidos de variação anatômica (oito pacientes), sangramento incoercível (quatro pacientes) e empiema de vesícula (três pacientes). Na colecistopatia crônica, as principais causas foram anatomia obscura (16 casos) e aderência (14 casos), sendo que em dez casos as duas causas estavam associadas. A diferença entre as duas formas da colecistopatia em relação a esta distribuição é significativa $(p=0,008)$. Enquanto na forma crônica a presença de anatomia obscura é vista em $80 \%$ dos casos, na forma aguda isso ocorre em pouco mais de $28 \%$ dos casos. Já a aderência é a causa de 46,42\% de conversão nos casos da doença na forma aguda e de apenas $20 \%$ na forma crônica (Tabela 1).

\section{DISCUSSÃO}

A colecistectomia videolaparoscópica esta relacionada à diminuição da dor no pós-operatório, menor período de internamento, retorno precoce às atividades e melhor resultado cosmético ${ }^{9}$. Além disso, estudos avaliando a resposta fisiológica, metabólica e bioquímica do corpo confirmam uma redução no trauma deste procedimento ${ }^{14-}$ 16. A colecistectomia laparoscópica é considerada padrão ouro para tratamento da colecistopatia litiásica sintomática desde $1987^{17}$.

A maioria das contra-indicações para como obesidade mórbida, cirurgia abdominal prévia e colecistite aguda não são mais consideradas contra-indicações absolutas. No entanto, a hipertensão arterial sistêmica e as desordens de coagulação constituem contra-indicações para o procedimento ${ }^{18-20 .}$

A morbimortalidade desse procedimento é claramente conhecido 1,3,4,21,22. Estudos controlados e randomizados mostram que a colecistectomia videolaparoscópica é superior a minilaparoscopia ${ }^{23}$. Há, no entanto, uma elevada proporção de casos em que não é possível sua realização. Além disso, em alguns pacientes, quando se tem dificuldades técnicas, é necessária a conversão de cirurgia videolaparoscópica para via aberta ${ }^{8}$. Esta conversão é feita em 1,5 a 19\% dos pacientes, devido, principalmente, à dificuldade anatômica e complicações inerentes à dissecção laparoscópica ${ }^{1-5}$.

Prakash et al. referem uma taxa de 56,5\% de conversão dos pacientes com colecistite com evidências de líquido Peri-colecístico no ultra-som pré-operatório ${ }^{10}$. Já a taxa de conversão do estudo de Eldar et al. foi de $40 \%$ dos pacientes com colecistite gangrenosa ${ }^{24}$.

Brodsky et al. estudando 956 pacientes submetidos à colecistectomia videolaparoscópica em Israel, encontraram uma taxa de conversão de 20,5\% (44 casos) $)^{7}$. Em um estudo de 1000 pacientes que sofreram colecistectomia laparoscópica, Kama et al. observaram 48 casos de conversão ${ }^{8}$.

Simopoulos et al. analisando 1804 pacientes de colecistectomia videolaparoscópica observaram que em 94 pacientes (2,8\%) foram necessários conversão. Destas, 44 foram por colecistite crônica e 50 por aguda 6 . A taxa de conversão também predominou na colecistite aguda (15,6\% dos 1212 pacientes) em comparação à crônica (4\%) no estudo realizado no ano de 2000 por Suter et al. ${ }^{12}$.

Tabela 1 - Causas de conversão de colecistectomia videolaparoscópica para cirurgia aberta nas colecistopatias agudas e crônicas.

\begin{tabular}{lrcc}
\hline & $\begin{array}{c}\text { Colecistopatia } \\
\text { aguda(n=28) }\end{array}$ & $\begin{array}{c}\text { Colecistopatia } \\
\text { crônica* }(n=20)\end{array}$ \\
\hline Aderência & 13 & $(46,42 \%)$ & $14(20,00 \%)$ \\
Anatomia Obscura & 8 & $(28,57 \%)$ & $16(80,00 \%)$ \\
Sangramento & 4 & $(14,28 \%)$ & - \\
Empiema de vasícula & 3 & $(10,71 \%)$ & - \\
\hline
\end{tabular}

(*) Dez pacientes (50\%) apresentaram mais de uma causa para conversão. 
No presente estudo encontrou-se uma taxa de conversão geral de $4,5 \%$, sendo que, destes, $7,58 \%$ foram em colecistite aguda e 2,85\% em colecistite crônica. Em relação às causas de conversão cirúrgica, destacaram-se a dificuldade anatômica $(59,25 \%)$ na forma crônica e a presença de aderências $(68,42 \%)$ na aguda.

A Taxa de conversão aceitável para pacientes com colecistopatia crônica é de $3-5 \%{ }^{1,25}$, já para a aguda é de $6-35 \%{ }^{26,27}$.
Nos trabalhos revisados e assim como neste observam-se dois fatos em comum. Primeiro, a taxa de conversão cirúrgica em colecistectomias predomina nas colecistopatias agudas quando comparadas com a doença na forma crônica. Segundo, as principais causas que levam à necessidade de conversão são: presença de grande quantidade de aderências, por inflamação intensa (principalmente nas colecistopatias agudas); e dificuldade de identificação das estruturas anatômicas e dissecção do Triângulo de Calot (principalmente nas colecistopatias crônicas).

\title{
A B S T T R A C T
}

\begin{abstract}
Objectives: to analyze the rate and main causes for conversion to open surgery among acute and chronic cholecystitis. Methods: a retrospective analysis of 1359 patient medical charts submitted to cholecystectomy in hospital universitário cajuru's general surgery department was done during the period from january 2000 to october 2006. Results: one thousand and sixty six laparoscopic cholecystectomies were performed, 701 (65.75\%) due to chronic cholecystolithiasis, 356 (33.39\%) due to acute cholecystolithiasis, seven $(0.65 \%)$ had gallbladder polyps and two $(0.21 \%)$ cases had hydropic gallbladder. The conversion rate in acute cases was $7.86 \%$, and in chronic cases were $2.85 \%$ ( $p=0.0003$ ). The average age for conversion was $50.96 \pm 17.49$ years-old for acute cases and $56.45 \pm 12.28$ years-old for chronic cases. The operative meantime was 152.5 minutes (30 - 36$)$ for acute and 157.5 minutes $(90-240)$ for chronic cholecystolithiasis $(p=0.959)$. The conversion reasons for chronic cholecystolithiasis were: obscure anatomy in 16 cases (80\%) and adhesions in 14 cases (70\%), considering that in ten cases both causes were associated. For acute presentation, conversions were due to adhesions in 13 (46.42\%) patients and technical difficulties were found in $8(28.57 \%)$ patients $(p=0.008)$. Conclusion: the rate for conversion of laparoscopic cholecystectomy to open surgery is higher in acute cholecystolithiasis when in comparison to chronic ones. Both, the presence of adhesions in acute cases, and difficult anatomy identification in chronic cases, were the most common reasons to impair the laparoscopic procedures performance in our department.
\end{abstract}

Key words: Cholecystectomy. Cholecystitis, acute. Chronic Disease.

\section{REFERENCIAS}

1. Cushieri A, Dubois F, Mouiel J, Mouret P, Becker H, Buess G, et al. The European experience with laparoscopic cholecystectomy. Am J Surg. 1991; 161(3):385-7.

2. Larson GM, Vitale GC, Casey S, Evans JS, Gilliam G, Heuser L, et al. Multipractice analysis of laparoscopic cholecystectomy in 1,983 patients. Am J Surg. 1992; 163(2):221-6.

3. Scott TR, Zucker KA, Bailey RW. Laparoscopic cholecystectomy: a review of 12,391 patients. Surg Laparosc Endosc. 1992; 2(3):1918.

4. The Southern Surgeons Club. A prospective analysis of 1,518 laparoscopic cholecystectomies. N Engl J Med. 1991; 324(1):10738. Erratum in: N Engl J Med 1991; 325(21):1517-8.

5. Steele RJ, Marshall K, Lang M, Doran J. Introduction of laparoscopic cholecystectomy in a large teaching hospital: independent audit of the first 3 years. Br J Surg. 1995; 82(7):968-71.

6. Simopoulos C, Botaitis S, Polychronidis A, Tripsianis G, Karayiannakis AJ. Risk factors for conversion of laparoscopic cholecystectomy to open cholecystectomy. Surg Endosc. 2005; 19(7):905-9. Epub 2005 May 4.

7. Brodsky A, Matter I, Sabo E, Cohen A, Abrahamson J, Eldar S. Laparoscopic cholecystectomy for acute cholecystitis: can the need for conversion and the probability of complications be predicted? Surg Endosc. 2000; 14(8):775-60.

8. Kama NA, Doganay M, Dolapci M, Reis E, Atli M, Kologlu M. Risk factors resulting in conversion of laparoscopic cholecystectomy to open surgery. Surg Endosc. 2001; 15(9):965-8. Epub 2001 Jun 12.

9. Alponat A, Kum CK, Koh BC, Rajnakova A, Goh PM. Predictive factors for conversion of laparoscopic cholecystectomy. World J Surg. 1997; 21(6): 629-33.

10. Prakash K, Jacob G, Lekha V, Venugopal A, Venugopal B, Ramesh H. Laparoscopic cholecystectomy in acute cholecystitis: Factors associated with conversion. Surg Endosc. 2002; 16(1):180-3. Epub 2001 Oct 5.
11. Wang YC, Yang HR, Chung PK, Jeng LB, Chen RJ. Urgent laparoscopic cholecystectomy in the management of acute cholecystitis: timing does not influence conversion rate. Surg Endosc. 2006; 20(5):806-8. Epub 2006 Mar 16.

12. Suter M, Meyer A. A 10 years experience with the use of laparoscopic cholecystectomy for acute cholecystitis: is it safe? Surg Endosc. 2001; 15(10):1187-92. Epub 2001 Aug 16.

13. Kitano S, Matsumoto T, Aramaki M, Kawano K. Laparoscopic cholecystectomy for acute cholecystitis. J Hepatobiliary Pancreat Surg. 2002; 9(5):534-7.

14. Aktan $A O$, Büyükgebiz $O$, Ye $\square$ en $C$, Yalin R. How minimally invasive is laparoscopic surgery. Surg Laparosc Endosc. 1994; 4(1):18-21.

15. Cho JM, LaPorta AJ, Clark JR, Schofield KJ, Hammond SL, Mallory $\mathrm{PL}$. Response of serum cytokines in patients undergoing laparoscopic cholecystectomy. Surg Endosc. 1994; 8(12):1380-3; discussion 1383-4.

16. Mealy K, Gallagher H, Barry M, Lennon F, Traynor O, Hyland J. Physiological and metabolic responses to open and laparoscopic cholecystectomy. Br J Surg. 1992; 79(10):1061-4.

17. Dubois F, Berthelot G, Levard H. Coelioscopic cholecystectomy: experience with 2006 cases. World J Surg. 1995; 19(5):748-52.

18. Frazee RC, Roberts JW, Symmonds R, Snyder SK, Hendricks J, Smith $R$, et al. What are the contraindications for laparoscopic cholecystectomy? Am J Surg. 1992; 164(5):491-4; discussion 494-5.

19. Hodgson WJ, Mercan S, Morgan J. Laparoscopic cholecystectomy in a tertiary referral center. Surg Laparosc Endosc. 1993; 3(3):2048.

20. Miles RH, Carballo RE, Prinz RA, McMahon M, Pulawski G, Olen $\mathrm{RN}$, et al. Laparoscopy: the preferred method of cholecystectomy in the morbid obese. Surgery. 1992; 112 (4):818-22; discussion 822-3.

21. Deziel DJ, Millikan KW, Economou SG, Doolas A, Ko ST, Airan MC. Complications of laparoscopic cholecystectomy: a national survey of 4,292 hospitals an analysis of 77,604 cases. Am J Surg. 1993; 165(1):9-14. 
22. Wolfe BM, Gardiner BN, Leary BF, Frey CF. Endoscopic cholecystectomy. An analysis of complications. Arch Surg. 1991; 126(10):1192-6; discussion 1196-8.

23. Barkun JS, Barkun AN, Sampatis JS, Fried G, Taylor B, Wexler MJ, et al. Randomised controlled trial of laparoscopic versus mini cholecystectomy. The McGill Gasllstone Treatment Group. Lancet. 1992; 340(8828):1116-9.

24. Eldar S, Sabo E, Nash E, Abrahamson J, Matter I. Laparoscopic cholecystectomy for the various types of gallbladder inflammation: a prospective trial. Surg Laparosc Endosc. 1988; 8(3):200-7.

25. Berci G, Sackier JM. The Los Angeles experience with laparoscopic cholecystectomy. Am J Surg. 1991; 161(3):382-4.

26. Lai PB, Kwong KH, Leung KL, Kuok SP, Chan AC, Chung SC, Lau WY. Randomized trial of early versus delayed laparoscopic cholecystectomy for acute cholecystitis. Br J Surg. 1998; 85(6)764-7.

27. Rattner DW, Ferguson C, Warshaw AL. Factors associated with successful laparoscopic cholecystectomy for acute cholecystitis. Ann Surg. 1993; 217(3):233-6.
Recebido em 28/08/2008

Aceito para publicação em 13/11/2008

Conflito de interesse: nenhum

Fonte de financiamento: nenhuma

\section{Como citar este artigo:}

Von Bahten LC, Isa AC, Campezato H, Figueiredo PC, Isa RH, Olandoski M. Colecistopatia aguda e crônica: análise comparativa das taxas e causas de conversão para cirurgia aberta. Rev Col Bras Cir. [periódico na Internet] 2009; 36(2). Disponível em URL: http://www.scielo.br/rcbc

\section{Endereço para correspondência:}

Ana Cristina Isa

Email: icrisana@yahoo.com.br 